\title{
OBTENCIÓN DE MODELOS PARA LA DETERMINACIÓN DE LA EVAPOTRANSPIRACIÓN DE REFERENCIA EN CONDICIONES DE CLIMA SUBHÚMEDO SECO EN LAS TUNAS, CUBA
}

\author{
GETTING MODELS TO DETERMINE THE REFERENCE \\ EVAPORATION-PERSPIRATION UNDER SUBHUMID-DRY \\ WEATHER CONDITIONS IN LAS TUNAS, CUBA
}

MsC. Leyder Santana Peña ; DrC. Esteban Peña Peña ${ }^{2}$

\begin{abstract}
RESUMEN
Para estimar la evapotranspiración de referencia (ETo) en la zona norte de Las Tunas en Cuba, se realizó un estudio en el que se comparó su dinámica en la zona y se comparó con la evapotranspiración potencial (ETp) medida en lisímetro. Se emplearon los métodos de Penman-Monteith (PM), Turc (T), Hargreaves Samani (HS), Thorntwaite (Th), Blaney Criddle (BC) y Blaney Morin (BM). Las fórmulas que mejor reflejaron el comportamiento real de la evapotranspiración de referencia en el norte de Las Tunas fueron la de Hargreaves Samani, Penman Monteith, Turc y Blaney Morin. Se obtuvo una alta correlación directa entre la evapotranspiración de referencia calculada y las variables del clima: temperatura media, insolación y evaporación. Se obtuvieron ocho ecuaciones de regresión para el cálculo de la evapotranspiración de referencia, factibles de poder utilizarse en el cálculo de las necesidades hídricas de los cultivos en las condiciones del norte de Las Tunas.

Palabras clave: Evapotranspiración; evapotranspitación de referencia.
\end{abstract}

\begin{abstract}
To estimate the reference evaporation-perspiration (ETo) in the north area of Las Tunas in Cuba, it was carried out a study about its dynamics and was compared with the potential evaporation-perspiration (ETP) measure in lysimeter. There were used the methods of Penman-Monteith (PM), Turc (T), Hargreaves Samani (HS), Thorntwaite (Th), Blaney Criddle (BC) and Blaney Morin (BM). The equations that better reflected the real behavior of the reference evaporation-perspiration in the north of The Tunas were those of Hargreaves Samani, Penman Monteith, Turc and Blaney Morin. A high direct correlation was obtained among the evaporation-perspiration of calculated reference and the variables of the climate: half temperature, heatstroke and evaporation. Eight regression equations were obtained for the calculation of the reference evaporation-perspiration, feasible of being able to be used in the calculation of the hydrics necessities of the crops under the conditions of the north of Las Tunas.

key words: Evaporation-perspiration, Referente Evaporation-perspiration.
\end{abstract}

\section{INTRODUCCIÓN}

Cuba está trabajando en la formación de una conciencia de la naturaleza en la población y ha desarrollado planes de producción de alimentos utilizando diferentes alternativas que por un lado satisfagan las necesidades de la población y por otro que constituyan verdaderos sistemas sostenibles. No tener un conocimiento sobre las condiciones hídricas de una región ha causado en muchos lugares del mundo grandes desastres agrícolas. Con técnicas nada complejas es posible hacer estudios de

1 Profesor Auxiliar, Facultad de Agronomía, Centro de Estudios de Desarrollo Agrario en Las Tunas. Centro Universitario de Las Tunas.Cuba. leyder@ult.edu.cu

2 Profesor Titular, Centro de Estudios de Desarrollo Agrario en Las Tunas. Facultad de Agronomía. Centro Universitario de Las Tunas. Cuba. Dirección postal Avenida Carlos J. Finlay s/n Reparto Buenavista, Las Tunas. Cuba. Código 75200. esteban@ ult.edu.cu 
éstas y planificar estrategias agrícolas que ayuden a evitar equívocos y obtener resultados sostenibles (Vázquez, 2003).

El cálculo de la evapotranspiración es el primer paso para establecer las necesidades de riego de los cultivos, y en él se unen dos procesos energéticos, evaporación y transpiración, mediante los cuales se produce transferencia de vapor a la atmósfera (Penman, 1948).

En condiciones tropicales el cálculo del balance hídrico hecho a partir de fórmulas de evapotranspiración presenta diferencias apreciables con él obtenido a partir de mediciones de humedad en el suelo. Entre otras cosas, estas diferencias se deben a que tales métodos no son representativos para aquellas condiciones, al haberse desarrollado en latitudes templadas. Por este motivo se considera conveniente disponer de una ecuación más adaptable al trópico.

El presente trabajo se efectuó con el objetivo de estudiar la dinámica de la evapotranspiración de referencia (ETo) que refleje el comportamiento real del proceso en las condiciones del norte de Las Tunas que puedan ser utilizadas con fines de planificación y explotación del riego.

\section{MATERIALES Y MÉTODOS}

Se realizó un estudio de la dinámica de la evapotranspiración de referencia en la región norte de la provincia Las Tunas, obteniéndose mediante regresión ecuaciones regionales para el cálculo de la misma en las condiciones de la zona.

Se trabajó con siete fórmulas de cálculo de la evapotranspiración de referencia (ETo) corrientemente empleadas en el país y a su vez de las más citadas por la literatura: Penman Monteith, Hargreaves Samani, Turc, García-López, Thornthwaite, Blaney Criddle y Blaney Morin.

Los datos climatológicos utilizados fueron los promedios mensuales del período 1975-2002 (28 años), provenientes de la Estación Meteorológica de Puerto Padre.

Se aplicó la metodología de Martelo (1989) para comparar las tasas de evapotranspiración de referencia calculadas por las fórmulas anteriores con la obtenida por métodos aerodinámicos y lisimétricos (ETp) referida por ACC (2002).

La comparación se realizó por medio de tres índices:
1. Índice Estacional (IE)

2. Relación Porcentual(RP)

3. Coeficiente de Correlación (r)

Una vez calculada la ETp mediante las siete fórmulas y habiéndose estimado los tres índices para cada una de ellas, se procedió a su jerarquización mediante un sencillo sistema de pesos asignados de manera arbitraria. A la que mejor ajuste tenía en cada índice se le asignaron 7 puntos y así en orden decreciente hasta la de menor ajuste. Luego se sumó la puntuación obtenida en cada índice y se consideraron importantes sólo aquellas fórmulas que aparecieron ubicadas en los primeros cuatro lugares. De esta manera pudo elegirse con mayor objetividad la fórmula mejor adaptada.

Después de elegir la mejor fórmula para cada estación se procedió a correlacionar la ETp así calculada con las variables del clima a fin de comprobar cuál o cuáles eran las que mayor influencia tenían sobre el poder evaporante de la atmósfera, resultando la evaporación y la temperatura las que mayor correlación mostraron con la ETo, procediéndose entonces a calcular ecuaciones de regresión entre cada fórmula original y las mencionadas variables, obteniéndose así 8 ecuaciones de regresión con un buen ajuste (dos por cada fórmula original).

\section{RESULTADOS Y DISCUSIÓN}

Según se observa en el Cuadro 1, los índices estacionales oscilaron entre 8,3 y 16,4. Las fórmulas Penman Monteith (PM) y Hargreaves Samani (HS) alcanzaron los mejores valores, seguidas de Blaney Morin (BM) y Turc (T). En cuanto al coeficiente de correlación, éste osciló entre 0,78 y 0,92 y los resultados no difirieron mucho respecto al aspecto anterior, siendo de nuevo las cuatro fórmulas mencionadas las que también ocuparon los primeros lugares, repitiéndose el mismo orden. Al analizar la relación porcentual, ésta varió entre 68 y 97,9 y se observaron algunas diferencias en comparación con los dos índices analizados, aunque tres (BM, PM y HS) de las cuatro fórmulas señaladas estuvieron en los primeros lugares, siendo García López (GL) la cuarta de mejor posición en este caso.

De las siete fórmulas probadas, cuatro se seleccionaron como mejor adaptadas de acuerdo a la puntuación final creciente que logró cada una. Éstas fueron por orden jerárquico: PM, HS, BM y T. 
La fórmula de Penman Monteith se derivó de la de Penman de 1948, y tanto ésta como la de Turc, han sido mencionadas por Rey et al. (1978) con posibilidades de ser aplicadas para el cálculo de la evapotranspiración de referencia, porque ambas se adaptan bien a climas húmedos como el de Cuba, pero tienen el inconveniente práctico de que necesitan una gran cantidad de datos, por lo que se ven limitadas para ser aplicadas a nivel local, desde el punto de vista de la poca disponibilidad de éstos.

De acuerdo al criterio de los mismos autores, las fórmulas que en este estudio arrojaron valores inadecuados para ser seleccionadas (Blaney Criddle y Thorntwaite) tienen el inconveniente de que se basan en la temperatura, y esta variable por sí sola no es buena indicadora del poder evaporante de la atmósfera.

Criterios más actuales sostenidos por Jensen (1990) señalan las fórmulas de Penman Monteith y Hargreaves Samani con buenas posibilidades para ser empleadas en el cálculo de la evapotranspiración de referencia en el trópico, con desviaciones que oscilaron entre $\pm 1 \%$ y $\pm 4 \%$ respecto a la evapotranspiración obtenida en lisímetros instalados en esas condiciones, mientras Loggia et al. (2002) señalaron a Hargreaves Samani con buenas posibilidades de aplicación, pues los inconvenientes que presenta el que utilice la temperatura como indicador de la evapotranspiración se corrigen con la introducción del término $\mathrm{Ra}$ (radiación equivalente en $\mathrm{mm} /$ día), el que depende de la latitud del lugar y refleja mejor las condiciones locales.

En el Cuadro 2 se observa la alta correlación directa existente entre los valores calculados por las cuatro mejores fórmulas con la temperatura, la insolación y la evaporación. Las fórmulas de $\mathrm{T}$ y HS, mientras BM y PM se correlacionaron mejor con la evaporación. Todas mostraron una muy débil relación inversa con la humedad relativa y se diferenciaron respecto a su relación con la velocidad del viento, pues en el caso de PM se observa una muy débil correlación inversa con esta variable, mientras las restantes presentaron correlaciones directas significativas.

Los valores del coeficiente de correlación para la temperatura, aunque son menores que los alcanzados con la variable Insolación y Evaporación, también son altos, por lo que se puede afirmar que las tres variables de conjunto influyen directamente sobre la magnitud del poder evaporante que se alcance en una localidad. Estos resultados coinciden con lo planteado en la literatura al respecto, pues en estudios reportados por Acosta y Luis (1986); Jensen, et al. (1990); Smith, et al. (1990) y Allen, et al. (1998) se destaca la gran influencia que provocan estas tres variables, siendo las causantes principales de los volúmenes de agua que entran a la atmósfera desde el suelo, ya sea por la vía de la evaporación o de la transpiración.

A partir de los resultados analizados se procedió a buscar las ecuaciones de regresión mediante las cuales fuera posible obtener, para cada una de las cuatro seleccionadas, una fórmula local que relacionara las tasas de ETo calculadas con las variables del clima y poder utilizarse en el futuro con fines de predicción de las necesidades hídricas de los cultivos en el ambiente subhúmedo seco del norte de Las Tunas.

\section{Cuadro 1}

Comparación e índices utilizados para determinar la fórmula de ETo mejor adaptada al norte de Las Tunas

\begin{tabular}{|l|c|c|c|c|c|c|c|}
\hline \multicolumn{1}{|c|}{ Fórmula } & IE & Poscón & R & Poscón & RP & Poscón & Puntuación general \\
\hline García López & 11,7 & 3 & 0,85 & 2 & 77,3 & 4 & 9 \\
\hline Thornthwaite & 16,4 & 1 & 0,78 & 1 & 68,0 & 1 & 3 \\
\hline Turc & 9,9 & 4 & 0,89 & 4 & 78,8 & 3 & 11 \\
\hline Hargreaves-Samani & 8,7 & 6 & 0,904 & 6 & 76,7 & 5 & 17 \\
\hline Penman-Monteith & 8,3 & 7 & 0,92 & 7 & 76,2 & 6 & 20 \\
\hline Blanney Morin & 9,1 & 5 & 0,901 & 5 & 73,6 & 7 & 17 \\
\hline Blanney Criddle & 12,6 & 2 & 0,85 & 3 & 97,9 & 2 & 7 \\
\hline
\end{tabular}


De esta forma se lograron ocho ecuaciones con posibilidades de ser utilizadas, las que se relacionan a continuación (Cuadro 3), así como su coeficiente de determinación. Como se aprecia los coeficientes de determinación fueron altos en todos los casos, oscilando entre 0,85 y 0,98 , lo que refleja un buen ajuste entre las variables.

Donde Ev y T son la evaporación mensual $(\mathrm{mm})$ y la temperatura promedio mensual para una localidad.

\section{Cuadro 2}

Relación entre las variables climáticas y los valores de ETo calculados por las fórmulas de mejor adaptación a la zona norte de Las Tunas

\begin{tabular}{|l|c|c|c|c|c|}
\hline \multirow{2}{*}{\multicolumn{1}{|c|}{ Fórmula }} & \multicolumn{5}{|c|}{ Coeficiente de correlación } \\
\cline { 2 - 6 } & Temperatura & Humedad Relativa & Velocidad del viento & Insolación & Evaporación \\
\hline Turc & 0,83 & $-0,04$ & 0,66 & 0,96 & 0,93 \\
\hline Hargreaves-Samani & 0,80 & $-0,09$ & 0,65 & 0,94 & 0,77 \\
\hline Penman-Monteith & 0,73 & $-0,14$ & $-0,05$ & 0,75 & 0,97 \\
\hline Blanney Morin & 0,67 & 0,02 & 0,48 & 0,95 & 0,98 \\
\hline
\end{tabular}

Cuadro 3

Ecuaciones de regresión obtenidas

\begin{tabular}{|l|l|c|c|}
\hline \multicolumn{1}{|c|}{ Fórmula base } & \multicolumn{1}{c|}{ Ecuación } & No. Ecuación & R $^{\mathbf{2}}$ \\
\hline Penman Monteith & $\mathrm{ETo}=11,9+0,613 * \mathrm{Ev}$ & 1 & 0,95 \\
\hline Penman Monteith & $\mathrm{ETo}=-51,9+0,538^{*} \mathrm{Ev}+3,08 * \mathrm{~T}$ & 2 & 0,90 \\
\hline Turc & $\mathrm{ETo}=33,2+0,517 * \mathrm{Ev}$ & 3 & 0,87 \\
\hline Turc & $\mathrm{ETo}=-83,2+5,61 * \mathrm{~T}+0,38 * \mathrm{Ev}$ & 4 & 0,98 \\
\hline Hargreaves Samani & $\mathrm{ETo}=-177+10,1 * \mathrm{~T}+0,286 * \mathrm{Ev}$ & 5 & 0,88 \\
\hline Hargreaves Samani & $\mathrm{ETo}=-241+14,7 * \mathrm{~T}$ & 6 & 0,97 \\
\hline Blaney Morin & $\mathrm{ETo}=-10,4+1,27 * \mathrm{~T}+0,537 * \mathrm{Ev}$ & 7 & 0,97 \\
\hline Blaney Morin & $\mathrm{ETo}=16,1+0,568 * \mathrm{Ev}$ & 8 & 0,85 \\
\hline
\end{tabular}

\section{CONCLUSIONES}

Las fórmulas que mejor reflejaron el comportamiento real de la evapotranspiración de referencia en el norte de Las Tunas fueron la de Hargreaves Samani, Penman Monteith, Turc y Blaney Morin.

Se obtuvo una alta correlación directa entre la evapotranspiración de referencia calculada y las variables del clima temperatura media, insolación y evaporación.

Se obtuvieron ocho ecuaciones de regresión para el cálculo de la evapotranspiración de referencia, factibles de poder utilizarse en el cálculo de las necesidades hídricas de los cultivos en las condiciones del norte de Las Tunas. 


\section{LITERATURA CITADA}

A.C.C. 2002. Centro Provincial de Meteorología Las Tunas. Informe del comportamiento de las variables del clima en la provincia Las Tunas, Folleto impresión ligera.

ACOSTA, F.; LUIS, A. 1986. Estudio de la evapotranspiración en caña de azúcar. INACA, Riego y Drenaje: 2(2): 22-61.

ALLEN R.; PEREIRA, L.; RAES, D.; SMITH, M. 1998. Crop evapotranspiration: guidelines for computing crop water requirements. Rome: FAO, Irrigation Drainage Pág. 56.

JENSEN, M.; BURMAN, R.;ALLEN, R. 1990. Evapotranspiration and Irrigation Water Requirements. ASCE Manuals and Reports on Engineering Practice $N^{\circ} 70.332 \mathrm{pp}$

LOGGIA, F.; PENSINI, M.; SARDO, V. 2005. HargreavesSamani method and evaporation pan in the estimation of reference evapotranspiration. ISHS Acta Horticulturae 449: II international symposium on irrigation of horticultural crops, 2002. Disponible en http://www.actahort.org/. Consulta mayo.
MARTELO, MARÍA. 1989. Selección de la fórmula de evapotranspiración mejor adaptada al área de Guanare-Masparro. Rev. Fac. Agron. (Maracay), 15: 185-206.

PENMAN, H.L. 1948. Natural evaporation from open water, bare soil and grass. Proc. R. Soc. London, Ser. A. 193: 120-145.

REY, R.; DE LA HOZ, L.; STINCER, J. 1978. Cálculo de la evapotranspiración de los cultivos agrícolas. Método del Evaporímetro Clase A. Ciencia y Técnica en la Agricultura, Riego y Drenaje. Vol I, No 1 . Enero.

SMITH M.; ALLEN, R.; MONTEITH, J.L.; PERRIER, A.; SANTOS PEREIRA, L. 1990. Report on the expert consultation on procedures for revision of FAO guidelines for prediction of crop water requirements. Reunión FAO, Roma. 28-31 mayo.

VÁZQUEZ, R. 2003. Desarrollo sostenible. Ojeada agrometeorológica en el contexto de la sostenibilidad agrícola. Congreso Internacional de Meteorología, La Habana (Formato electrónico). 